\title{
Preface to the Proceedings of the European General Assembly on IHY 2007
}

\author{
C. Briand · E. Antonucci · H. J. Haubold
}

Received: 24 October 2008/ Accepted: 24 October 2008/Published online: 10 January 2009

(C) Springer Science+Business Media B.V. 2009

The year 2007 marks the 50th Anniversary of the International Geophysical Year (IGY) and 50 years of space exploration. The world science community has brought together an international program of scientific collaboration: the International Heliophysical Year (IHY) 2007-2008. IHY extends the concept of "Geophysics" to "Heliophysics" and deals not only with atmospheric and solar-terrestrial physics but also with studies of other planets, the outer reaches of the heliosphere, and its interaction with the interstellar medium.

The European coordination of IHY fosters the development of scientific programmes as well as outreach activities. IHY is also a good opportunity for the whole heliospheric community to settle on a new basis for future collaborations.

This special issue is based on a meeting that took place in Torino, Italy, in June 2007. One aim of the meeting was to foster European participation and collaboration in the development of the large infrastructures of the future for the exploration and the study of the heliosphere. Space- and ground-based major instruments that are presently in their initial design phase have been presented as support for discussions and contacts between scientific teams all over Europe. It was also an opportunity to present new ideas or projects. This meeting was particularly timely with respect to the ASTRONET ${ }^{1}$ efforts to establish a

\footnotetext{
1 ASTRONET was created by a group of European funding agencies in order to establish a comprehensive long-term planning for the development of European astronomy. The objective of this effort is to consolidate and reinforce the world-leading position that European astronomy has attained at the beginning of this $21 \mathrm{st}$ century. ASTRONET is financed by the European Community.
}

C. Briand

Observatoire de Meudon, LESIA - BAT 16, 5 place J. Janssen, F-92195 Meudon CEDEX Principal, France

e-mail: carine.briand@obspm.fr

E. Antonucci

INAF - Osservatorio di Torino, Via osservatorio 20, I-10025 Pino Torino, Italy

e-mail: antonucci@to.astro.it

H. J. Haubold $(\bowtie)$

UN Office for Outer Space Affairs, Vienna International Centre, 1400 Vienna, Austria

e-mail: hans.haubold@unvienna.org 
long-term planning for the development of European astronomy. It was also excellent timing for the presentation of the missions in the frame of the ESA Cosmic Vision call for proposal.

This special issue of Earth, Moon, and Planets thus reports on the state-of-art of the heliosphere exploration. In each session invited papers presented the scientific challenges of the next decade. These are then followed by descriptions of the instruments that are proposed to meet these challenges. A specific effort was made to bring together the spaceand ground-based communities concerned with all the heliospheric domains: the Sun, the solar wind, solar wind-planets relationships, space weather, and the limits of the heliosphere. Most large projects are described. In addition, two specific papers present the viewpoints of NASA and ESA in heliophysics studies and collaboration between the two agencies. We expect that this special issue will become a reference book about future instrument developments and will be of interest to a broad community.

The papers in this volume underwent a rigorous refereeing process to insure scientific quality and clarity. The overall reviewing and editing was done by myself (Hans Haubold), and A.M. Mathai (Canada, India). The referees for the ten papers from the UN/ES/NASA workshop were N. Gopalswamy (United States), T. Sakurai (Japan), G. Eichhorn (United States), and myself. Referees for the 28 papers from the Second European General Assembly were C. Briand (France), E. Antonucci (Italy), and myself. 